\title{
Stability analysis of the carbuncle phenomenon and the sonic point glitch
}

\author{
AAYUSH AGRAWAL ${ }^{1}$ and BALAJI SRINIVASAN ${ }^{2, *}$ \\ ${ }^{1}$ Department of Mechanical Engineering, Indian Institute of Technology Delhi, New Delhi 110016, India \\ ${ }^{2}$ Department of Applied Mechanics, Indian Institute of Technology Delhi, New Delhi 110016, India \\ e-mail: aayush.28agrawal@gmail.com; balaji@am.iitd.ac.in
}

MS received 29 November 2015; revised 31 August 2016; accepted 30 November 2016

\begin{abstract}
Upwinding allows for accurate, non-oscillatory capturing of shocks waves; however, many Riemann solvers (both exact and approximate) suffer from some sort of numerical instability. One of the most mysterious and least understood of these is the carbuncle phenomenon. In the present study, we analyse the closely allied "simplified carbuncle" problem, also known as the $2 \mathrm{D}$ shock stability problem or the $1.5 \mathrm{D}$ carbuncle problem. Motivated by the existence of some recently derived schemes that do not exhibit the instability, we perform a thorough stability analysis and extend previous studies by analysing the pseudo-spectra and hence the effects of non-normality in causing this instability. Our results establish that, contrary to previous indications in the literature, a non-linear mechanism is responsible for the instability. In order to understand the nature of this non-linear mechanism better, we perform a non-linear analysis of the sonic glitch, which shares some common features with the carbuncle. We provide two previously unknown results. Firstly, we show that even the "entropy-satisfying" Godunov scheme violates the entropy condition in the sonic glitch. Secondly, we provide a more accurate definition for the entropy condition for scalar conservation laws that supports the previous claim. We conjecture that a similar non-linear anti-dissipative mechanism might be responsible in triggering the carbuncle. This work is expected to lead to a better understanding of possible unphysical behaviour in Riemann solvers and thus help in the design of better solvers for high-Reynolds-number flows.
\end{abstract}

Keywords. Carbuncle phenomenon; sonic glitch; instability; non-normality; pseudo-spectra.

\section{Introduction}

The field of Computational Fluid Dynamics (CFD), i.e., the use of numerical methods for the solution of the governing equations of fluid flow, is now a mature field with a dazzling range of applications from weather prediction to the design of re-entry vehicles. However, despite the range of successes, there remain some fundamental questions about the robustness of the underlying discretization methods, especially in untested domains. Compressible flows, in particular, throw up a range of unexpected glitches, oscillations and outright unphysical numerical solutions, especially in parameter regimes where some physics transition happens. For instance, problems tend to occur at low Mach numbers, in the transonic regime and in the hypersonic regime. One of the first systematic catalogue of such failings of Riemann solvers was given by Quirk [1]. Ever since, many more such failings have been discovered [2-5] and serious attempts are being made by the wider research community to find solutions to such failings and design robust methods.

*For correspondence
One such failing that has attracted significant attention due to its mystifying and recalcitrant nature is the carbuncle phenomenon [6]. This problem occurs while computing high Mach number flows for a blunt body where some lowdiffusion schemes (most famously, Roe's scheme) converge to a completely unphysical solution as if there were a spike in front of the blunt body, instead of giving a clean detached bow shock. The fact that the physically incorrect solution is stable and is exhibited by even entropy-satisfying schemes such as Godunov is one of the primary enduring mysteries of this problem. Enticing as the mystery is, we note that it is not only for the mystery that the carbuncle problem is important. It is being actively investigated (see [7] and [8] for two among many recent examples), also because of its practical relevance. While solving for hypersonic flow past blunt bodies such as reentry vehicles, the potential presence of the carbuncle forces us to use fixes or overly dissipative schemes for the convective fluxes even in Navier-Stokes solvers [8]. These fixes, in turn, have the effect of adversely affecting the accuracy of heat-transfer predictions [9], which affect our ability to design Thermal Protection Systems (TPS) for these vehicles. 
The carbuncle phenomenon is not restricted to inviscid solvers and occurs even in Navier-Stokes solutions [10]. Recently, however, there has been evidence that the use of Navier-Stokes solvers along with grids fine enough to resolve the viscous shocks could eliminate these problems [11]. However, this is not a practical approach due to the enormous number of grid points that would be required for such resolution. As estimated in [12], for complete viscous resolution, even for a laminar, two-dimensional flow the number of computational cells required would be on the order of 108 , while $3 \mathrm{D}$ computations could require as many as 1012 cells, rendering such well-resolved viscous computations impractical. This is also the reason why the causes and cures for the carbuncle are still being investigated in the vanishing viscosity limit of Euler equations. The idea behind such inviscid investigations is that the additional artificial diffusion terms that various schemes offer are a proxy for modelling the effects of under-resolution of the viscous terms, especially around shocks.

It was in this inviscid context that Peery and Imlay [6] observed unphysical solutions using Roe's scheme for a blunt body problem and named this the carbuncle phenomenon due to the "blister-like shock profile" that appears instead of the bow shock. Quirk [1] offered a simpler version of the problem and ever since a large number of cures and conjectures for this phenomenon have been offered in the literature. The most popular one is the use of Harten's entropy fix [13] to modify the flux function, which results in increase in viscosity in transverse direction. Another cure proposed by Dumbser et al [14] is a transverse velocity component that can damp the transverse perturbations. Some authors have proposed to locally apply a dissipative scheme by flagging the cells in the vicinity of shock waves but this requires prior knowledge of solutions. Despite the large number of proposed cures (we refer the reader to Garicano-Mena et al [7] for a recent, comprehensive list), all cures are somewhat ad-hoc in nature and tend to add dissipation, whose effects on the final flow-field are not entirely clear.

The finding of cures has been simultaneously accompanied by attempts to diagnose the reasons for the carbuncle. Several conjectures and some theorems exist. Quirk [1] was the first to propose the explanation for the onset of carbuncle, which he attributed to the "odd-even decoupling" of pressure and density fields along the shock being out of phase with each other. The underlying reason for this decoupling was conjectured by Liou [15] to be due to multidimensional shock instability triggered by schemes in which the dissipation in the mass flux depends on the pressure term. While Liou's conjecture has been useful in developing cures for the carbuncle, (the RoeM1 and RoeM2 schemes of kim et al [16] were developed based on the conjecture), many contradictions of the conjecture have been found in literature. As noted by Pandolfi and D'Ambrosio [10], Kim et al [16] and Robinet et al [17], the
AUSM scheme, in some cases, may develop shock instability even though its mass flux is independent of the pressure term. The LRS scheme [18] also violates the Liou's conjecture. Similarly, EFM [19] and EIM [20] schemes have the same mass flux, but in violation of the conjecture, EFM does not produce the carbuncle while EIM does.

While Liou's conjecture remains popular due its simplicity, other explanations and analyses have been offered $[5,10,14,16]$. Due to the difficulty in analysing the original blunt-body problem, almost all of these analyses start from a closely allied problem-the 2D shock stability problem. The problem involves the analysis of a normal shock in a 2D grid subject to perturbations. These perturbations may be either due to the grid (as in the formulation introduced by Quirk [1]) or through upstream perturbations as in its latter simplified form due to Dumbser et al [14]. It has been observed that all problems that exhibit the blunt body carbuncle also exhibit stability problems in this simplified problem. This shock stability problem is therefore known as the "simplified carbuncle? or the " $1.5 \mathrm{D}$ carbuncle? [2].

Apart from its ease of analysis, we feel that there is a deeper reason that one ought to pay attention to the simplified carbuncle problem. In a recent paper, Elling [12] points out that the blunt-body carbuncle can be produced even physically by carefully introduced perturbations or "filaments upstream of the body". This means that the blunt-body carbuncle is a physical solution to a slightly perturbed problem. Since, in principle, it is never possible to completely eliminate such perturbations, Elling pessimistically concludes that "the carbuncle is incurable" (indeed, that is the title of his paper). While we, and others [8], disagree with his conclusion, an important corollary of Elling's work is that the simplified carbuncle problem does offer a better, less ambiguous setting. This is because, it is well known that a normal shock is stable to 2D perturbations and hence any scheme exhibiting an unstable shock is unambiguously unphysical. Therefore, for its close relation with the blunt-body carbuncle, its ease of analysis and its lack of ambiguity, we analyse the simplified carbuncle in the rest of this paper.

The simplified carbuncle was first analysed by Quirk [1], who presented a linear stability analysis of the oddeven decoupling phenomenon by taking perturbations in the density and pressure fields. Pandolfi and D'Ambrosio [10] showed that carbuncle phenomenon affects schemes that, when solving the Riemann problem, explicitly take into account the presence of a contact surface. These analyses were extended by Gressier and Moschetta [21] through a proper matrix stability analysis. One of the primary conclusions of this analysis was Gressier's theorem that strict stability on the simplified carbuncle test and exact resolution of contact discontinuity are incompatible. There were, however, two lacunae in this study. Firstly, the study was purely a modal, linear stability 
analysis, even though some of the phenomena exhibited by the shock (such as growth of perturbations and restabilization) were suggestive of a non-normal mechanism. Secondly, as shown by Srinivasan et al [18], Gressiers theorem is violated by the Linearized Riemann State (LRS) scheme, which is free of the carbuncle and also preserves the contact discontinuity. Further, as shown by Srinivasan et al [18], LRS is also analytically close to the Roe scheme, especially in the linearized regime. Our current study is therefore, motivated by the following questions. Firstly, is a non-normal instability mechanism involved in the carbuncle? Secondly, in case the instability mechanism is not non-normal but non-linear, can something be said about how even entropy-satisfying schemes such as Godunov end up producing this instability?

The rest of the paper is organized as follows. In section 2, we present a detailed stability analysis of the simplified carbuncle. The novelty here is that apart from the linear and matrix stability analysis we also present nonmodal stability analysis. Further, we analyse two schemes that do not show the carbuncle-Roe's scheme with Hartens fix and the LRS. We conclude that a non-linear instability mechanism is involved in the carbuncle. Section 3 tries to determine the reasons why entropy-satisfying schemes such as the Godunov could produce such instabilities through a non-linear analysis of the sonic glitch in Burgers equation using the Godunov, LRS and Roe's scheme. Finally, in section 4, the major conclusions that can be drawn from the study are presented.

\section{Stability analysis of the simplified carbuncle problem}

\subsection{The simplified carbuncle problem}

As mentioned in the previous section, analysis of the complete carbuncle problem is complicated due to the geometry. Therefore, following a standard approach in the literature first introduced by Quirk [1] and later modified by Gressier and Moschetta [21] and Sanders et al [22], we present an analysis of a version of the problem, with simplified geometry. This problem, variously known as the shock stability problem, simplified carbuncle or the $1.5 \mathrm{D}$ carbuncle problem, involves a stationary, normal shock placed in a two-dimensional domain and subject to upstream perturbations.

The details of the problem are as follows:

1. The domain is a $2 \mathrm{D}$ regular Cartesian mesh without any grid-line perturbations (which are present in Quirk's version of the test).

2. The domain extends from 0 to 1 in both directions ( $x$ and $y$ ) and is divided into $N \times N$ cells (we follow the literature in taking $25 \times 25$ cells).
3. A steady shock parallel to the $y$-axis is located at the centre with upstream and downstream conditions obtained from the Rankine-Hugoniot jump relations.

4. Boundary conditions in the $x$-direction are imposed using ghost cells while in the $y$-direction, periodic boundary conditions are imposed.

5. Perturbations are added upstream of the shock at about $10^{-6}$ times the values of the upstream variables.

It has been observed in the literature that all problems that exhibit the full carbuncle also exhibit instability to small perturbations in this simplified carbuncle case. In fact, for high Mach numbers, Roe's scheme even exhibits spontaneous instability without any explicit addition of perturbations. In the following, we perform a thorough stability analysis of this problem. The purpose for the analysis is to determine if the causes behind the carbuncle can be understood by analysing schemes that are analytically close to each other but show varying behaviours on the carbuncle. We therefore concentrate primarily on three schemes:

1. The baseline Roe scheme. This has no adjustable parameters and shows instability on the simplified carbuncle problem.

2. Roe's scheme with Harten's fix [13]. This has an adjustable parameter and its stability on the simplified carbuncle problem depends on the adjustable parameter.

3. The LRS scheme. This scheme has no adjustable parameters and shows stability on the simplified carbuncle problem. Further, it is analytically close to the Roe scheme [18].

The reason for primarily concentrating our analysis to these three schemes is that their fluxes are close analytically but the ensuing behaviour on the carbuncle varies widely, offering us a possibility of gleaning the reasons for the carbuncle.

For each of these schemes, we provide analysis using three approaches, in order of increasing complexity and detail:

1. linear, local stability analysis;

2. modal matrix stability analysis;

3. non-modal matrix stability analysis.

As we show later, while each analysis explains some behaviour, there are important behaviours that are left out even by the non-modal stability analysis.

\subsection{Linear stability analysis}

The Euler equations governing the motion of inviscid flows can be written as

$$
\frac{\partial \rho}{\partial t}+\nabla \cdot(\rho \mathbf{u})=0
$$




$$
\begin{gathered}
\frac{\partial(\rho \mathbf{u})}{\partial t}+\nabla \cdot(\mathbf{u} \times(\rho \mathbf{u}))+\nabla p=0 \\
\frac{\partial\left(\rho e_{T}\right)}{\partial t}+\nabla \cdot\left(\mathbf{u}\left(\rho e_{T}+p\right)\right)=0 .
\end{gathered}
$$

In the following study, only 2D flow is considered. For the purpose of computation of such flows using the FiniteVolume Method, these equations can be written concisely in conservation form as

$$
\frac{\partial \mathbf{U}}{\partial t}+\frac{\partial \mathbf{F}}{\partial x}+\frac{\partial \mathbf{G}}{\partial y}=0
$$

where $\mathbf{U}$ is the vector of conserved variable; $\mathbf{F}$ and $\mathbf{G}$ are the vector of flux along $x$ and $y$, respectively.

The linear stability analysis presented here follows, in the main, the approach given by Gressier and Moschetta [21].

In discretized form, 2D Euler equations can be written as

$$
\mathbf{U}_{i, j}^{n+1}=\mathbf{U}_{i, j}^{n}-\frac{\Delta t}{\Delta x}\left[\mathbf{F}_{i+\frac{1}{2}, j}-\mathbf{F}_{i-\frac{1}{2}, j}\right]-\frac{\Delta t}{\Delta y}\left[\mathbf{G}_{i, j+\frac{1}{2}}-\mathbf{G}_{i, j-\frac{1}{2}}\right] .
$$

The mean state vector is denoted as $\mathbf{U}_{0}$ with the subscript 0 denoting properties of mean flow. It is confirmed from the numerical experiments (see [1]), that odd-even decoupling occurs in transverse planes parallel to the shock; therefore, for linear stability analysis, the stream-wise fluxes can be assumed to be balanced, i.e., $\mathbf{F}_{i+\frac{1}{2}, j}-\mathbf{F}_{i-\frac{1}{2}, j}=0$. This is true even across the steady $2 \mathrm{D}$ shock. Therefore, only the transverse flux remains and the equation is simplified to

$$
\mathbf{U}_{i, j}^{n+1}=\mathbf{U}_{i, j}^{n}-\frac{\Delta t}{\Delta y}\left[\mathbf{G}_{i, j+\frac{1}{2}}-\mathbf{G}_{i, j-\frac{1}{2}}\right] .
$$

We subtract the mean state $\mathbf{U}_{0}$ from Eq. (6). Then, assuming that the states have perturbation such that $\delta \mathbf{U}_{i, j-1}=-\delta \mathbf{U}_{i, j}=\delta \mathbf{U}_{i, j+1}$, and linearizing the difference of transverse fluxes $\mathbf{G}_{i, j+\frac{1}{2}}-\mathbf{G}_{i, j-\frac{1}{2}}$, i.e., taking only the first order perturbation terms and neglecting the higher order terms $(\delta() . \delta() \simeq 0$ ), we can rewrite Eq. (6) as

$$
\delta \mathbf{U}_{j}^{n+1}=\delta \mathbf{U}_{j}^{n}-\frac{\Delta t}{\Delta y} \mathbf{G} \cdot \delta \mathbf{U}_{j}^{n}=\left(\mathbf{I}-\frac{\Delta t}{\Delta y} \mathbf{G}\right) \cdot \delta \mathbf{U}_{j}^{n}=\mathbf{A} \cdot \delta \mathbf{U}_{j}^{n} .
$$

For simplicity, the index $i$ has been dropped as we are concerned only with the transverse flux. The matrix $\mathbf{A}$ in this equation is the amplification matrix. For the scheme to remain stable to odd-even decoupling, the eigenvalues of amplification matrix must be less than 1. For eigenvalues greater than 1, the scheme would be unstable and marginally stable for eigenvalue equal to 1 . We now apply this analysis to Roe's scheme with and without Harten's fix and the LRS scheme. 2.2a Analysis of baseline Roe's scheme: The flux in Roe's scheme is given as

$$
\mathbf{G}_{i+\frac{1}{2}}=\frac{1}{2}\left(\mathbf{G}_{i}+\mathbf{G}_{i+1}\right)-\frac{1}{2} \sum_{k} \alpha_{j+\frac{1}{2}}^{k}\left|\lambda_{j+\frac{1}{2}}^{k}\right| \mathbf{R}_{j+\frac{1}{2}}^{k},
$$

where $\alpha_{j+\frac{1}{2}}^{k}$ are the wave strengths, $\lambda_{j+\frac{1}{2}}^{k}$ are the corresponding eigenvalues (characteristic speeds) and $\mathbf{R}_{j+\frac{1}{2}}^{k}$ are the corresponding right eigenvectors. From our assumptions it can be shown that

$$
\alpha_{j+\frac{1}{2}}^{k}=-\alpha_{j-\frac{1}{2}}^{k}, \lambda_{j+\frac{1}{2}}^{k}=\lambda_{j-\frac{1}{2}}^{k}, \mathbf{R}_{j+\frac{1}{2}}^{k}=\mathbf{R}_{j-\frac{1}{2}}^{k} .
$$

Using these, and doing some simplifications we get the following for Roe's scheme:

$$
\left(\begin{array}{l}
\delta \rho \\
\delta u \\
\delta v \\
\delta p
\end{array}\right)^{n+1}=\left[\begin{array}{cccc}
1 & 0 & 0 & -\frac{2 v}{a_{0}^{2}} \\
0 & 1 & 0 & 0 \\
0 & 0 & 1-2 v & 0 \\
0 & 0 & 0 & 1-2 v
\end{array}\right]\left(\begin{array}{c}
\delta \rho \\
\delta u \\
\delta v \\
\delta p
\end{array}\right)^{n}
$$

where $v=\frac{a_{0} \delta t}{\delta y}$ is the CFL number and $a_{0}=\sqrt{\frac{\gamma p_{0}}{\rho_{0}}}$ is the speed of sound.

The eigenvalues of the amplification matrix obtained are the terms on the leading diagonal of the matrix. It can be clearly seen that the stream-wise and transverse velocity perturbations are decoupled and that the stream-wise flux has the amplification factor of 1 , which makes it marginally stable. It can also be seen that although the pressure perturbation is stable, the density perturbation is not. We also note that the pressure perturbation feeds directly into the density perturbation, in line with Liou's conjecture.

2.2b Roe's scheme with Harten's fix: Roe's scheme by itself does not satisfy the entropy condition, and admits non-physical solutions like expansion shocks also. To cure this, Harten's entropy fix [13] is used with Roe's scheme. While the original fix was intended to address pathological entropy problems, it was later observed that Harten's fix, when applied to linear waves associated with shear and contact waves, cures the carbuncle. Unfortunately, there is still no clear understanding of any possible physical justification for this. Further, the fix also causes the smearing of contact discontinuity. Harten's entropy fix is given as

$$
|\lambda|=\left\{\begin{array}{cc}
|\lambda| & \text { if }|\lambda|>\delta \\
\frac{\delta^{2}+\lambda^{2}}{2 \delta} & \text { if }|\lambda| \leq \delta
\end{array}\right.
$$

where $\lambda$ is the eigenvalue or the characteristic speed and $\delta=\delta_{0}(|u|+|a|)$. A suitable value of $\delta_{0}$ is selected for the simulation. It is known from the numerical experiments that a threshold value of 0.2 is needed to cure the carbuncle 
[21]. Carrying out a similar analysis as that of Roe's scheme, we obtain

$$
\begin{aligned}
& \left(\begin{array}{l}
\delta \rho \\
\delta u \\
\delta v \\
\delta p
\end{array}\right)^{n+1} \\
& =\left[\begin{array}{cccc}
1-\frac{2 \delta_{0} v}{a_{0}} & 0 & 0 & -\frac{2 v}{a_{0}^{2}}\left(1-\frac{\delta_{0}}{a_{0}}\right) \\
0 & 1-\frac{2 \delta_{0} v}{a_{0}} & 0 & 0 \\
0 & 0 & 1-2 v & 0 \\
0 & 0 & 0 & 1-2 v-2 v \frac{\delta_{0}}{a_{0}} \frac{u^{2}}{a^{2}}(\gamma-1)
\end{array}\right] \\
& \times\left(\begin{array}{c}
\delta \rho \\
\delta u \\
\delta v \\
\delta p
\end{array}\right)^{n} \\
&
\end{aligned}
$$

Again, the eigenvalues of the amplification matrix are the diagonal terms of the matrix. The amplification factors of density and stream-wise velocity have become $1-\frac{2 \delta_{0} v}{a_{0}}$ instead of 1 and the amplification factor of pressure perturbation has significantly reduced. We see that because of the fix, the scheme has become stable as compared with marginal stability without the fix. However, we note an important subtlety that has gone unnoticed in the literature thus far; the amplification matrix obtained indicates that Roe's scheme with Harten's fix should become stable for even a slightly positive value of $\delta$, which is not in agreement with the numerical experiments. Later on in the paper, we analyse the results from a full fledged matrix stability analysis to see if they explain these results.

2.2c Analysis of the LRS scheme: The LRS scheme uses the concept of upwinding to find the state at the cell interface directly instead of the flux, i.e., the state $\mathbf{U}_{i+\frac{1}{2}}$ is found by approximately solving the Riemann problem and then the interface flux is calculated as

$$
\mathbf{F}_{i+\frac{1}{2}}=\mathbf{F}\left(\mathbf{U}_{i+\frac{1}{2}}\right)
$$

where

$$
\mathbf{U}_{i+\frac{1}{2}}=\frac{1}{2}\left(\mathbf{U}_{i}+\mathbf{U}_{i+1}\right)-\frac{1}{2} \sum_{k} \alpha_{i+\frac{1}{2}}^{k} \operatorname{sgn}\left(\lambda_{j+\frac{1}{2}}^{k}\right) \mathbf{R}_{j+\frac{1}{2}}^{k}
$$

When linear stability analysis is performed on LRS scheme, taking into account only the first-order perturbation terms, we get the following:

$$
\left(\begin{array}{c}
\delta \rho \\
\delta u \\
\delta v \\
\delta p
\end{array}\right)^{n+1}=\left[\begin{array}{cccc}
1 & 0 & 0 & -\frac{2 v}{a_{0}^{2}} \\
0 & 1 & 0 & 0 \\
0 & 0 & 1-2 v & 0 \\
0 & 0 & 0 & 1-2 v
\end{array}\right]\left(\begin{array}{c}
\delta \rho \\
\delta u \\
\delta v \\
\delta p
\end{array}\right)^{n}
$$

We note that the amplification matrix is the same as that obtained in Roe's scheme. Srinivasan et al [18] showed that LRS scheme is similar to Roe's scheme up to the first-order dissipative terms, which is reflected in the present analysis. It is noteworthy that that despite the equality of the local amplification matrices, Roe and LRS show widely different behaviours on the simplified carbuncle. One can therefore conclude immediately that the carbuncle in not a simple, one-dimensional local instability.

\subsection{Modal and non-modal matrix stability analysis}

Given the insufficiencies of the local linear stability analysis, we proceed to a full matrix stability analysis to see if it can explain the differences in the schemes above. The advantage a matrix stability analysis over the linear stability analysis performed earlier is that it examines instabilities triggered not only due to local amplification of perturbations in a single cell but also instabilities triggered due to the interaction of perturbations in different cells. Matrix stability analysis therefore offers the possibility of determining subtler causes of instability.

Mathematically, matrix stability analysis works by obtaining the rate of change of the perturbations vector with respect to the initial perturbation, i.e., if $\delta \mathbf{U}_{0}$ is the initial value of perturbation, then we need to find a matrix $\mathbf{S}$ such that,

$$
\frac{d}{d t}(\delta \mathbf{U})=\mathbf{S} . \delta \mathbf{U}_{0} .
$$

The matrix $\mathbf{S}$ accounts for the interaction of perturbations across various cells in the domain.

At this point, one may analyse merely the spectra of the matrix of the stability matrix or also its pseudospectra. In spectral or modal analysis, if the maximum real part of eigenvalue of the stability matrix $\mathbf{S}$ is positive, then the scheme will be unstable for the perturbations as the perturbation will amplify with time. If, however, the maximum real part of the eigenvalues is negative, then the perturbations will decay with time and thus the scheme would be stable. Marginal stability will be achieved if the real part of the eigenvalues is zero. Such an analysis is called modal stability analysis.

In the analysis of the stability of flows, it has been found that modal analysis fails to corroborate with the experimental results, an example of which is the stability of the wall-bounded viscous flows (Poiseuille flow and Couette flow), in which experiments give a much lower value of 
critical Reynolds number than that predicted by the stability analysis. The cause was initially attributed to some nonlinear mechanism. However, it was later recognized that the problem lay not with non-linearity but with non-normality, which this analysis cannot capture. Sometimes, the modes that are asymptotically stable may non-normally interact with each other and may lead to development of instability in short term. That is, two modes with a phase difference might constructively interfere in order to cause strong transient growth. This may occur whenever the stability matrix is non-normal. The work of Gustavsson [23], Butler and Farrell [24], Reddy et al [25], Reddy and Henningson [26] and Trefethen et al [27] establishes that perturbations in the viscous flows could grow by factor as large as 1000 and the growth occurs without any non-linear effects. The analogue of the eigenvalues for analysing non-normal operators/matrices is the pseudospectra (see section 2.3b). The role of non-normality in the development of carbuncle is studied in the paper.

The stability matrix that we obtain for the carbuncle problem is strongly non-normal; hence, we also augment this analysis through an analysis of the pseudospectra of various schemes as well. There is no existing non-modal analysis for the carbuncle problem in the literature to the authors' knowledge.

2.3a Formulation of the stability matrix: We follow here, in the main, the matrix stability approach of Dumbser et al [14]. We analyse the 2D steady shock problem for a structured mesh with a steady shock located at the centre of the domain and random perturbations being added to all flow variables. To analyse the stability of the finite-volume discretization, we first start with the integral form of the Euler equations given as

$$
\frac{d}{d t} \iiint_{V} \mathbf{U} d V+\iint_{A} \Psi \cdot \mathbf{n} d A=0
$$

$\iiint_{V} \mathbf{U} d V=\mathbf{U} V$ where $\mathbf{U}$ is the approximate cell average. Let $\mathbf{U}$ be the global vector of state and $\mathbf{Z}$ be the vector of state written in a local coordinate system. In the local coordinate system, the direction of flow is labelled as the local $x$-direction while in global coordinate system the axes are fixed to the usual Cartesian axes. Here, we use the rotational invariance property of Euler equations and write the flux term in the integral form of conservation equation as $\iint_{A} \mathbf{M} \psi(Z) d A$ where $\mathbf{M}$ is the rotation matrix, which relates the local and global state vectors as follows:

$$
\begin{gathered}
\mathbf{U}=\mathbf{M Z} \\
\mathbf{M}(\theta)=\left[\begin{array}{cccc}
1 & 0 & 0 & 0 \\
0 & \cos \theta & \sin \theta & 0 \\
0 & -\sin \theta & \cos \theta & 0 \\
0 & 0 & 0 & 1
\end{array}\right] .
\end{gathered}
$$

Therefore, the semi-discrete Euler equation can now be written as

$$
\frac{d \mathbf{U}_{i}}{d t}+\frac{1}{V_{i}} \sum_{k=1}^{4} M_{i k} \psi_{i k} A_{i k},
$$

where $V_{m}$ is the volume of the cell, $i$ refers to the cell under consideration, $k$ refers to the neighbouring cells, $A_{i k}$ is the area of the boundary between the cells $i$ and $k$ and $\psi_{i k}$ is the flux between cell $i$ and $k$. The flux function $\psi$ will depend on the scheme being used. As the mesh is structured and two dimensional, we have four neighbouring cells for each cell.

If we assume that the vector of state comprises a steady mean value $(\widehat{\mathbf{U}})$ and the perturbation in the cell $(\delta \mathbf{U})$, then the vector of state can be written as

$$
\mathbf{U}=\hat{\mathbf{U}}+\delta \mathbf{U} .
$$

The derivative of the flux with respect to the global vector of state in terms of local vector of state can be written as follows:

$$
\frac{\partial \psi_{i k}}{\partial U_{i, k}}=\frac{\partial \psi_{i k}}{\partial Z_{i, k}} \cdot \frac{\partial Z_{i, k}}{\partial U_{i, k}}=\frac{\partial \psi_{i k}}{\partial Z_{i, k}} \cdot M_{i k}^{-1} .
$$

Now, using the Taylor expansion of the flux function, taking the steady state as the mean state,

$$
\begin{gathered}
\psi_{i k}\left(Z_{i}, Z_{k}\right)=\psi_{i k}\left(M_{i k}^{-1} U_{i}, M_{i k}^{-1} U_{k}\right)=\psi_{i k}\left(M_{i k}^{-1} \hat{U}_{i}, M_{i k}^{-1} \hat{U}_{k}\right) \\
+\frac{\partial \psi_{i k}}{\partial Z_{i}} \cdot M_{i k}^{-1} \cdot \delta U_{i}+\frac{\partial \psi_{i k}}{\partial Z_{k}} \cdot M_{i k}^{-1} \cdot \delta U_{k} .
\end{gathered}
$$

Here, the derivative of the flux is calculated using the central difference as

$$
\frac{\partial \psi_{i}}{\partial Z_{j}}=\frac{\psi_{i}\left(Z_{j}+\Delta Z_{j}\right)-\psi_{i}\left(Z_{j}-\Delta Z_{j}\right)}{2 \Delta Z_{j}}, \quad i, j \leq 3
$$

where $\Delta Z_{j}$ can be suitably chosen. The analysis here is done taking $\Delta Z_{j}=10^{-6}$.

On combining Eqs. (16), (17) and (19), we get

$$
\begin{aligned}
\frac{d \delta U_{i}}{d t}= & -\frac{A_{i k_{1}}}{V_{i}} M_{i k_{1}} \frac{\partial \psi_{i k_{1}}}{\partial Z_{i}} M_{i k_{1}}^{-1} \cdot \delta U_{i}-\frac{A_{i k_{2}}}{V_{i}} M_{i k_{2}} \frac{\partial \psi_{i k_{2}}}{\partial Z_{i}} M_{i k_{2}}^{-1} \cdot \delta U_{i} \\
& -\frac{A_{i k_{3}}}{V_{i}} M_{i k_{3}} \frac{\partial \psi_{i k_{3}}}{\partial Z_{i}} M_{i k_{3}}^{-1} \cdot \delta U_{i}-\frac{A_{i k_{4}}}{V_{i}} M_{i k_{4}} \frac{\partial \psi_{i k_{4}}}{\partial Z_{i}} M_{i k_{4}}^{-1} \cdot \delta U_{i} \\
& -\frac{A_{i k_{1}}}{V_{i}} M_{i k_{1}} \frac{\partial \psi_{i k_{1}}}{\partial Z_{k_{1}}} M_{i k_{1}}^{-1} \cdot \delta U_{k_{1}}-\frac{A_{i k_{2}}}{V_{i}} M_{i k_{2}} \frac{\partial \psi_{i k_{2}}}{\partial Z_{k_{2}}} M_{i k_{2}}^{-1} \cdot \delta U_{k_{2}} \\
& -\frac{A_{i k_{3}}}{V_{i}} M_{i k_{3}} \frac{\partial \psi_{i k_{3}}}{\partial Z_{k_{3}}} M_{i k_{3}}^{-1} \cdot \delta U_{k_{3}}-\frac{A_{i k_{4}}}{V_{i}} M_{i k_{4}} \frac{\partial \psi_{i k_{4}}}{\partial Z_{k_{4}}} M_{i k_{4}}^{-1} \cdot \delta U_{k_{4}} .
\end{aligned}
$$

The first four terms on the right hand side of this equation give dependence of rate of change of error on the error in the cell itself, while the last four terms give the dependence of rate of change of error on the error in the neighboruing 
cells. We have a total of $n=N M$ cells, where $N$ and $M$ are the number of cells in the $x$ and $y$ direction, respectively. On combining all the cells, we obtain

$$
\frac{d}{d t}\left(\begin{array}{c}
\delta U_{1} \\
\delta U_{2} \\
\vdots \\
\delta U_{N} M
\end{array}\right)=S\left(\begin{array}{c}
\delta U_{1} \\
\delta U_{2} \\
\vdots \\
\delta U_{N} M
\end{array}\right)
$$

The solution of this equation is

$$
\left(\begin{array}{c}
\delta U_{1} \\
\delta U_{2} \\
\vdots \\
\delta U_{N M}
\end{array}\right)_{t=\tau}=e^{S \tau}\left(\begin{array}{c}
\delta U_{1} \\
\delta U_{2} \\
\vdots \\
\delta U_{N M}
\end{array}\right)_{t=0} .
$$

$\mathbf{S}$ in this equation is the desired stability matrix, which is of size $4 N M$. The modal stability of any scheme can be determined by the maximum real part of eigenvalues of the stability matrix. We now discuss how this stability matrix may also be used to determine the non-modal stability through an analysis of the pseudospectra.

2.3b Pseudospectra and non-modal stability: As mentioned earlier, pseudospectra are the non-modal analogue of eigenvalues for stability analysis. The non-modal growth of the perturbations is caused because of non-normality of the eigenvectors. Matrices or operators whose eigenvectors are orthogonal are known as"normal". All matrices A that satisfy $\mathbf{A} \mathbf{A}^{*}=\mathbf{A}^{*} \mathbf{A}$ are called as normal matrices, where $\mathbf{A}^{*}$ is the conjugate transpose of matrix $\mathbf{A}$. The superposition of non-orthogonal eigenvectors (associated with stable eigenvalues) may lead to short-term transient growth in the perturbations. In such case, the behaviour predicted by the eigenvalues is incorrect and pseudospectra can predict the correct behaviour. For example, the operators encountered in Rayleigh-Benard convection problem and Taylor-Couette problem are normal and the modal stability analysis in these cases matches with the experimental results, while the operators encountered in viscous flows such as Poiseuille and Couette flows are non-normal, leading to disagreement between experimental results and modal stability analysis. The stability matrix $\mathbf{S}$ (Eq. (21)) obtained after linearization of the Euler equations for the simplified carbuncle problem is highly non-normal; hence, we study the effect of non-normality during the onset of carbuncle.

We discuss here some equivalent definitions of pseudospectra. If $\mathbb{C}$ is the set of complex number, $N$ is the dimension of the eigenspace and $\|$. $\|$ denotes a norm on $\mathbb{C}^{N}$, then the pseudospectra of $\mathbf{A}$ can be defined as follows.

Definition 1 Let $\mathbf{A} \in \mathbb{C}^{N \times N}$ and $\epsilon$ be an arbitrary number such that $\epsilon>0$; then $\epsilon$-pseudospectrum $\sigma_{\epsilon}(\mathbf{A})$ of $\mathbf{A}$ is set $z \in \mathbb{C}$ such that $\left\|(z-\mathbf{A})^{-1}\right\|>\epsilon^{-1}$.
Here, $\|$. $\|$ denotes the two-norm $\|.\|_{2}$ and $\sigma(\mathbf{A})$ denotes the spectrum (set of eigenvalues) of A. Another equivalent definition of pseudospecta that relates to the eigenvalue perturbation is given as follows.

Definition 2 Let $\mathbf{A} \in \mathbb{C}^{N \times N}$ and $\epsilon$ be an arbitrary number such that $\epsilon>0$; then $\epsilon$-pseudospectrum $\sigma_{\epsilon}(\mathbf{A})$ of $\mathbf{A}$ is set $z \in \mathbb{C}$ such that $z \in \sigma(\mathbf{A}+\mathbf{E})$ for some $\mathbf{E} \in \mathbb{C}^{N \times N}$ such that $\|\mathbf{E}\|<\epsilon$.

In other words, it can be said that $\epsilon$-pseudospectrum is the set of eigenvalues of a perturbed matrix $\mathbf{A}+\mathbf{E}$ where $\|\mathbf{E}\|<\epsilon$. For normal matrices, the bound of pseudospectra around the eigenvalues of matrix $\mathbf{A}$ would be a circle with the eigenvalue as centre and radius equal to $\epsilon$. But for non-normal matrices, the bound of pseudospectra is not a circle and may take any shape depending on the level of non-normality. Clearly, the larger this variation, the more likely that the problem's instability is governed by non-normal behaviour.

\section{3c Results of modal and non-modal stability analysis:}

Using this formulation, we calculate the stability matrix for the simplified carbuncle problem for a range of schemes at various Mach numbers. In addition to the Roe scheme (with and without the entropy fix) and LRS schemes mentioned earlier, we also perform the stability analysis for a number of well-known schemes for which only modal results are currently available. A MATLAB code was developed for computing the stability matrix for various schemes. The stability matrix thus obtained was used for the calculation of both the spectra and pseudospectra. The spectra were computed using MATLAB's inbuilt routines while the pseudospectra were calculated using "Eigtool" [28].

Figures 1, 2, 3, and 4 show the spectra and pseudospectra of AUSM, Godunov, LRS and Roe scheme, respectively, at an upstream Mach number value of 7 . The maximum real part of the eigenvalues is shown above the plot of spectra. The $\epsilon$ pseudospectra are computed for the $\epsilon$ values $10^{-7}$ $10^{-2}$ and the colour bar shows the $\log (\epsilon)$ value. The results indicate that AUSM scheme is marginally stable and LRS scheme is stable and while Roe and Godunov schemes are unstable for flow with upstream Mach number 7. The results match with the numerical experiments. Marginal stability of AUSM scheme is confirmed even by numerical experiments as the perturbations remain stable at a particular value as seen in Gressier and Moschetta [21], where the Roe scheme was switched with AUSM scheme after a few time steps in Quirk's test. Unlike previous non-modal studies of other flow problems ([23-26] etc.), the pseudospectra are also near limits set by the spectra and no major amplification of the eigenvalues is seen due to the effect of non-normality.

We now examine the puzzling effect of Harten's fix. As mentioned before, linear stability analysis shows that an infinitesimal positive value of Harten's parameter can cure 


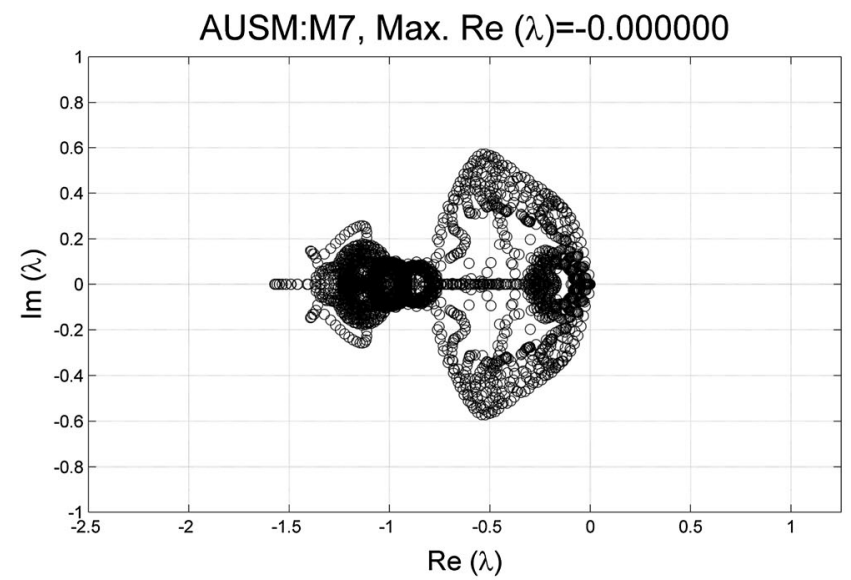

(a)

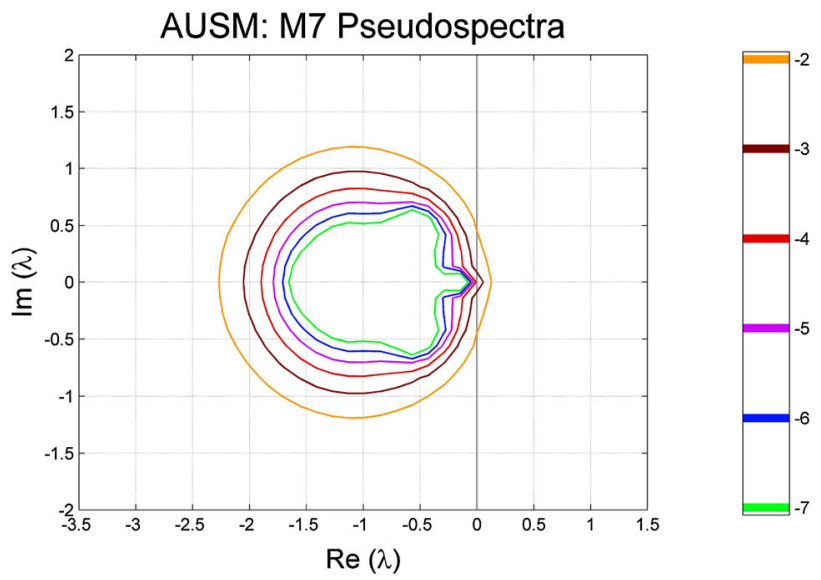

(b)

Figure 1. Figure showing the spectra and pseudospectra of the stability matrix of AUSM scheme at upstream Mach number 7. Colour bar on the right shows $\log (\epsilon)$. (a) AUSM scheme M7: spectra. (b) AUSM scheme M7: pseudospectra.

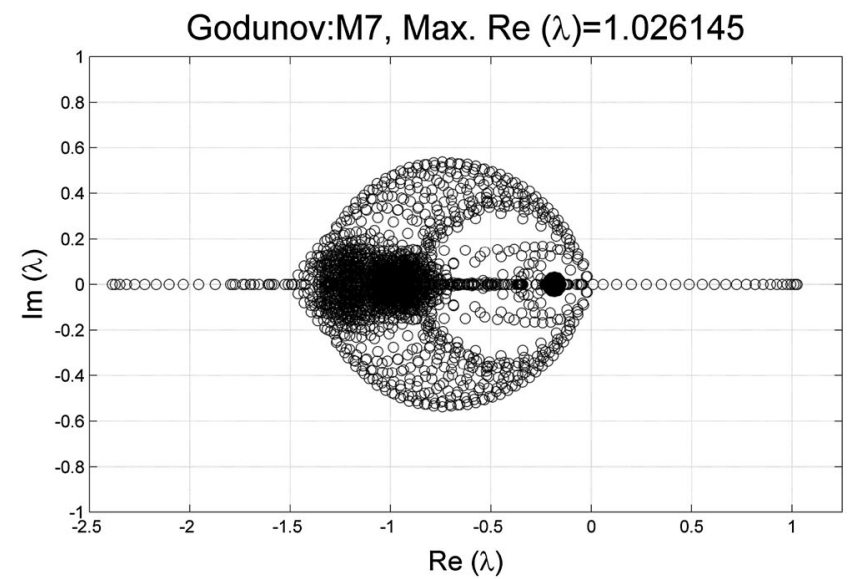

(a)

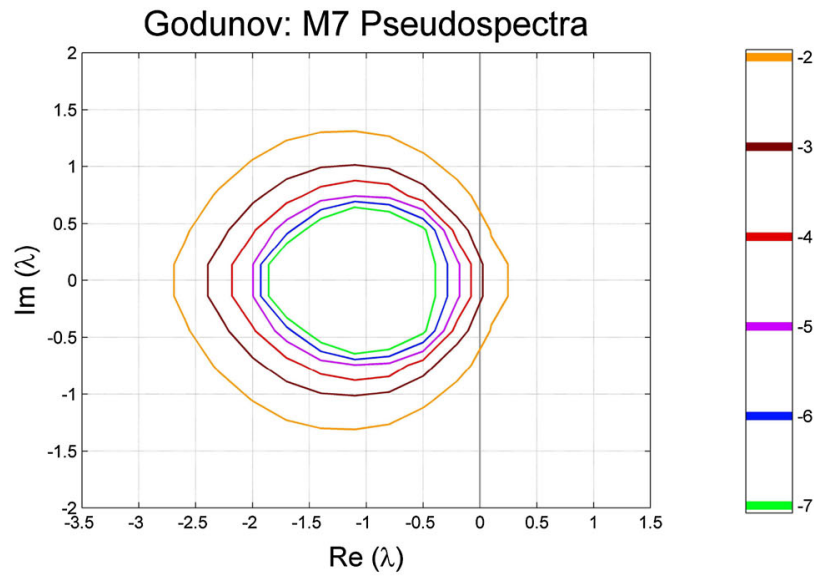

(b)

Figure 2. Figure showing the spectra and pseudospectra of stability matrix of Godunov scheme at upstream Mach number 7. Colour bar on the right shows $\log (\epsilon)$. (a) Godunov scheme M7: spectra. (b) Godunov scheme M7: pseudospectra.

the carbuncle while numerical experiments require a minimum finite value of 0.2 of Harten's parameter to completely cure the instability. To see if matrix stability analysis is able to provide any insight into this phenomenon, we perform both a modal and non-modal analysis of Roe's scheme with Harten's fix (henceforth called Roe-H scheme). Figures 5-7 show the spectra and pseudospectra of the Roe-H scheme with different $(0.2,0.5,2.5)$ values of Harten's parameter. Neither the modal or the non-modal stability curves are in agreement with the actual stability results from numerical experiments. The results show that even a large value of Harten's parameter is unable to cure the carbuncle. The pseudospectra are within limits and do not show strict stability even for the scheme where Harten's parameter greater than 0.2 is used.
Figure 8 shows the variation of maximum real part of eigenvalues of the stability matrix for the Roe- $\mathrm{H}$ scheme with the value of Harten's parameter. The variation is plotted for integer values of upstream Mach number from 2 to 10 . The results show that as the Harten's parameter is increased, the maximum of the real part of the eigenvalues decreases and if it crosses the right side of the imaginary plane, i.e., $\operatorname{Re}(\lambda)=0$, the scheme remains stable. For example, the scheme becomes stable at Mach 3 for Harten's parameter value of 0.5 , The maximum real part of eigenvalues decrease as value of Harten's parameter is increased and the scheme becomes stable at Harten's parameter value of 0.5 which is much higher than the actual experimental threshold of 0.2. However, for other cases with high Mach number, the maximum of the real part of eigenvalues decreases to a minimum positive value and then again starts 


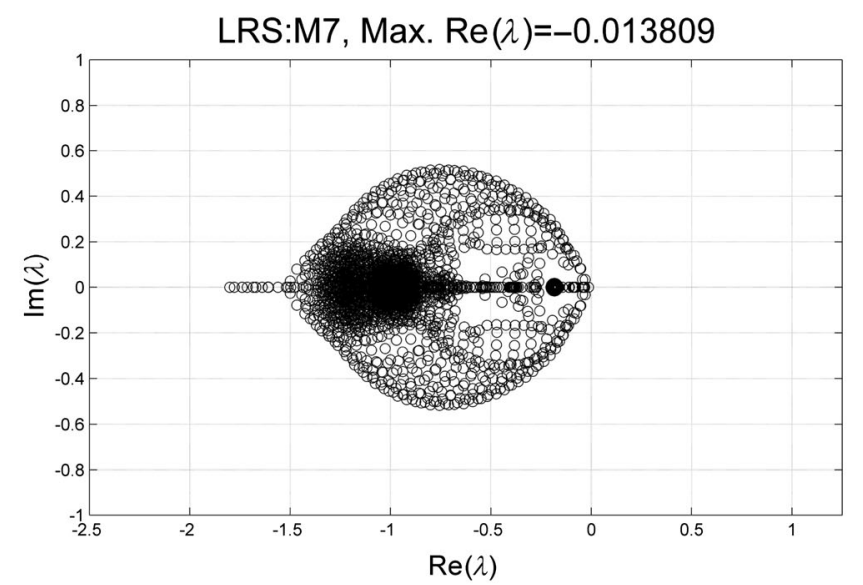

(a)

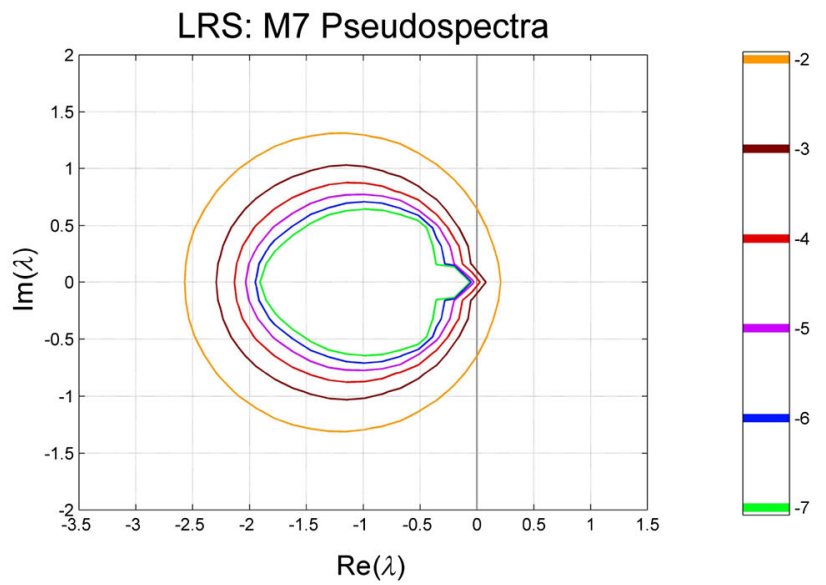

(b)

Figure 3. Figure showing the spectra and pseudospectra of the stability matrix of LRS scheme at upstream Mach number 7. Colour bar on the right shows $\log (\epsilon)$ (a) LRS scheme M7: spectra. (b) LRS scheme M7: pseudospectra.

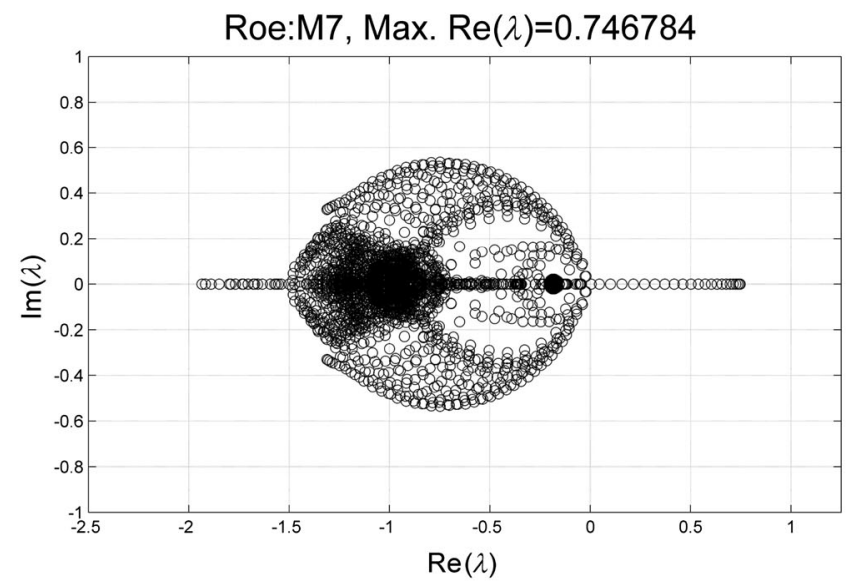

(a)

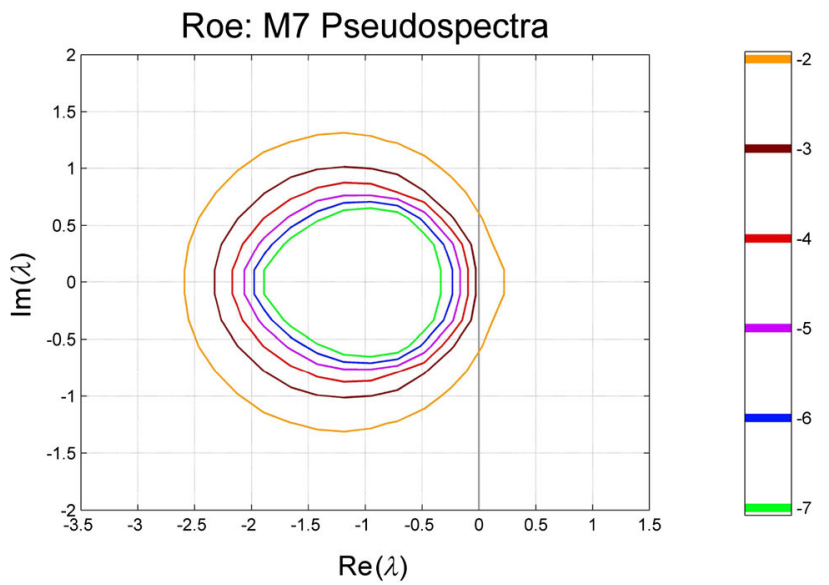

(b)

Figure 4. Figure showing the spectra and pseudospectra of the stability matrix of Roe scheme at upstream Mach number 7. Colour bar on the right shows $\log (\epsilon)$. (a) Roe scheme M7: spectra. (b) Roe scheme M7: pseudospectra.

increasing. The scheme can therefore be made stable only till an upstream Mach 7, after which no value of Harten's parameter can cure the scheme. This erratic behaviour adds more to the perplexing nature of the cure of carbuncle using the Harten's fix.

We now summarize the results of this section. Our primary purpose was to examine the instability mechanism in the carbuncle through a thorough analysis of three closely allied flux schemes, which nonetheless have significantly different behaviors on the carbuncle test. However, despite increasingly sophisticated linear stability tests-a local linear analysis and global modal analysis and global non-modal analysis, not all behaviours in the actual numerical tests corresponded to the theoretical stability analysis. In particular, the parameter range of Harten's fix obtained from the stability analysis is very different from what is obtained in numerical experiments and is used in practical codes. An ancillary result is the confirmation that non-normal modes play no role in the carbuncle instability.

While the result of this analysis seems negative, it does provide a clear future direction. Since no linear mechanism (local, global or non-modal) is able to account for the difference between Roe, Roe-H and LRS, there must therefore be an essentially non-linear mechanism that accounts for the carbuncle instability. Since we lack the tools for performing a non-linear stability analysis of this problem, we see instead if one may understand something about the nature of this non-linear mechanism by the analysis of a simpler problem that shows certain similarity in features. For this purpose, we analyse the sonic glitch in the next section. 


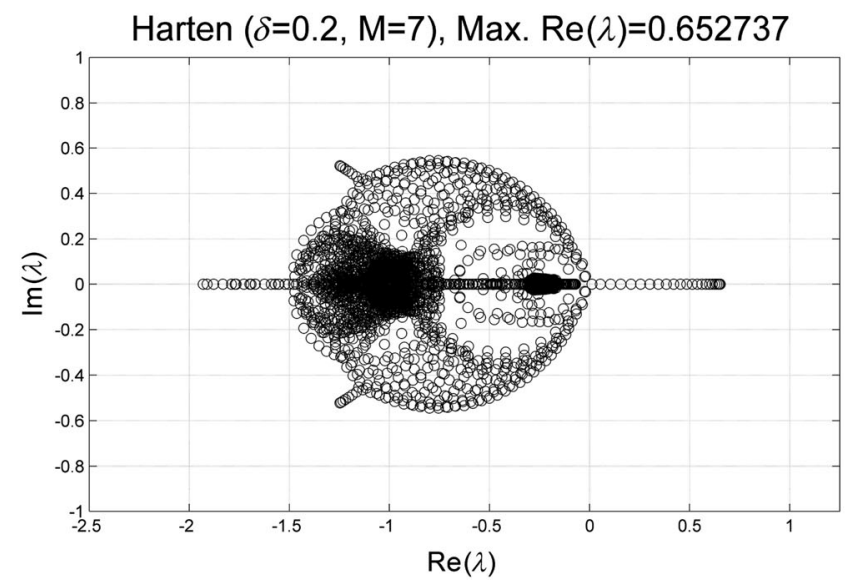

(a)

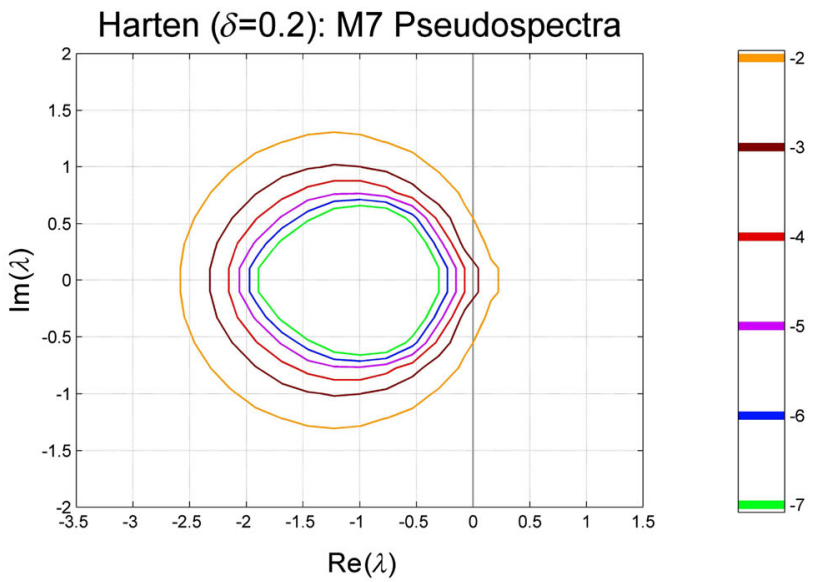

(b)

Figure 5. Figure showing the spectra and pseudospectra of the stability matrix of Roe-H scheme at upstream Mach number 7 and Harten's parameter 0.2. Colour bar on the right shows $\log (\epsilon)$. (a) $\operatorname{Roe}-\mathrm{H}(\delta=0.2, \mathrm{M} 7)$ : spectra (b) $\operatorname{Roe}-\mathrm{H}(\delta=0.2, \mathrm{M} 7)$ : $\operatorname{pseudospectra}$.

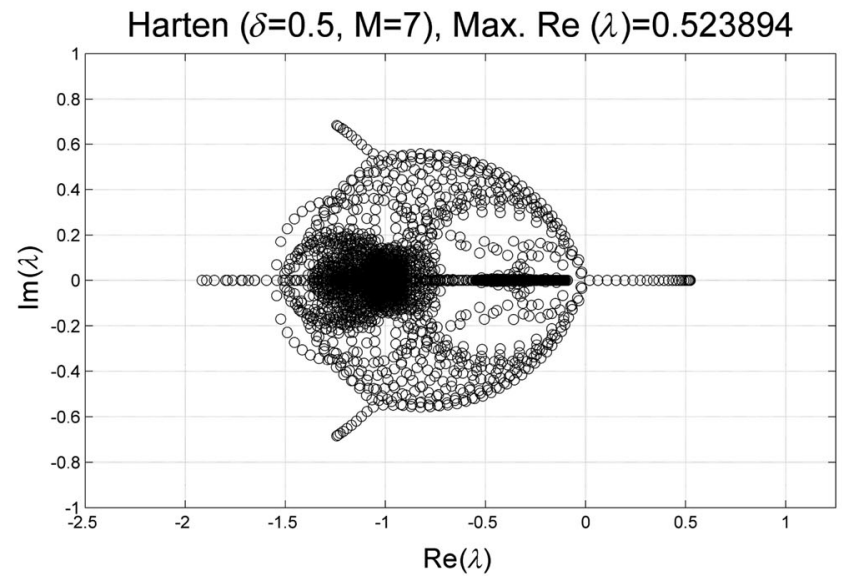

(a)

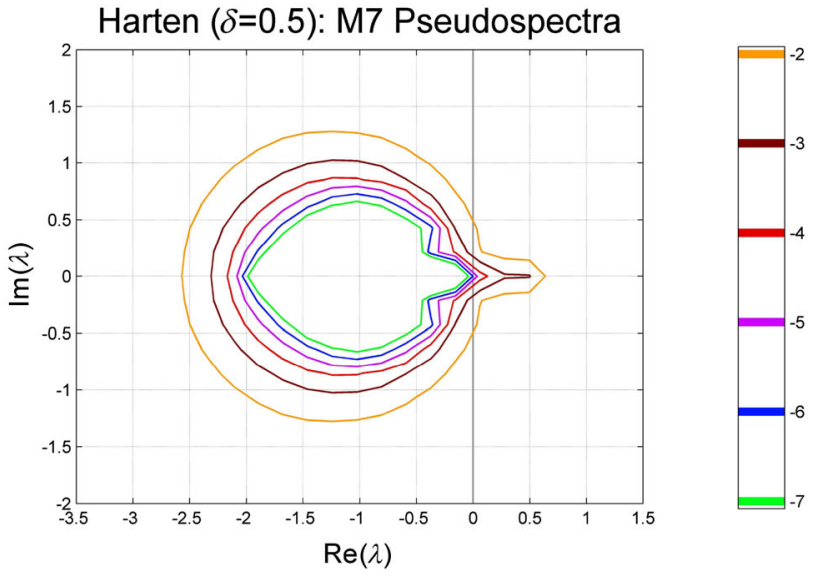

(b)

Figure 6. Figure showing the spectra and pseudospectra of the stability matrix of Roe-H scheme at upstream Mach number 7 and Harten's parameter 0.5. Colour bar on the right shows $\log (\epsilon)$. (a) $\operatorname{Roe}-\mathrm{H}(\delta=0.5$, M7): spectra (b) $\operatorname{Roe}-\mathrm{H}(\delta=0.5$, M7): pseudospectra.

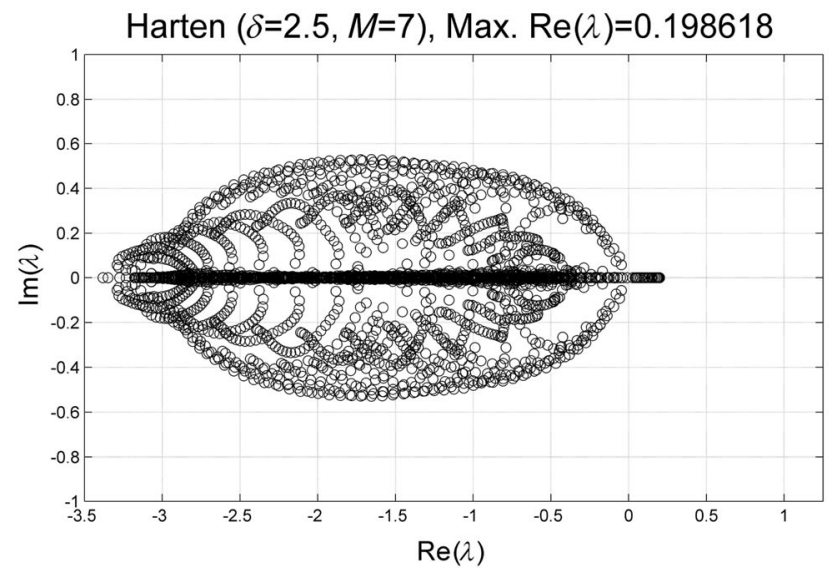

(a)

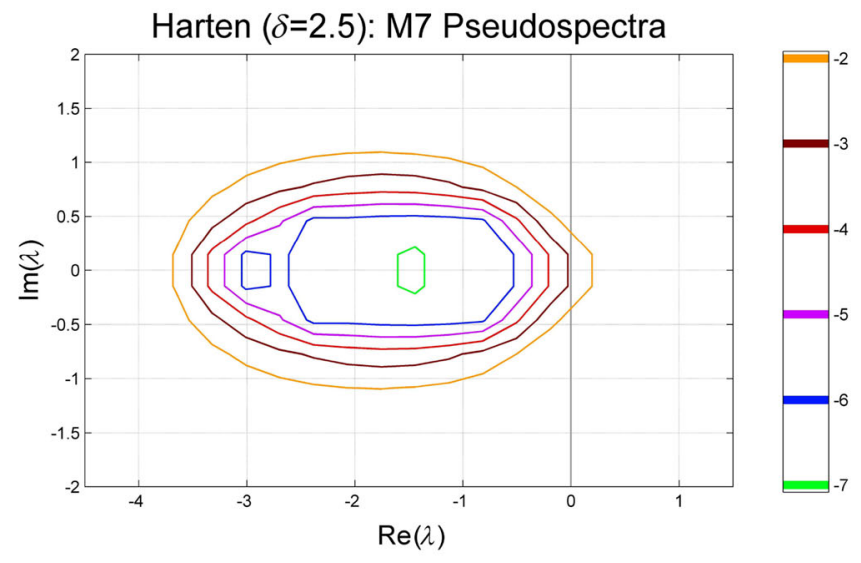

(b)

Figure 7. Figure showing the spectra and pseudospectra of the stability matrix of Roe-H scheme at upstream Mach number 7 and Harten's parameter 2.5. Colour bar on the right shows $\log (\epsilon)$. (a) $\operatorname{Roe}-\mathrm{H}(\delta=2.5$, M7): spectra (b) $\operatorname{Roe}-\mathrm{H}(\delta=2.5$, M7): pseudospectra. 


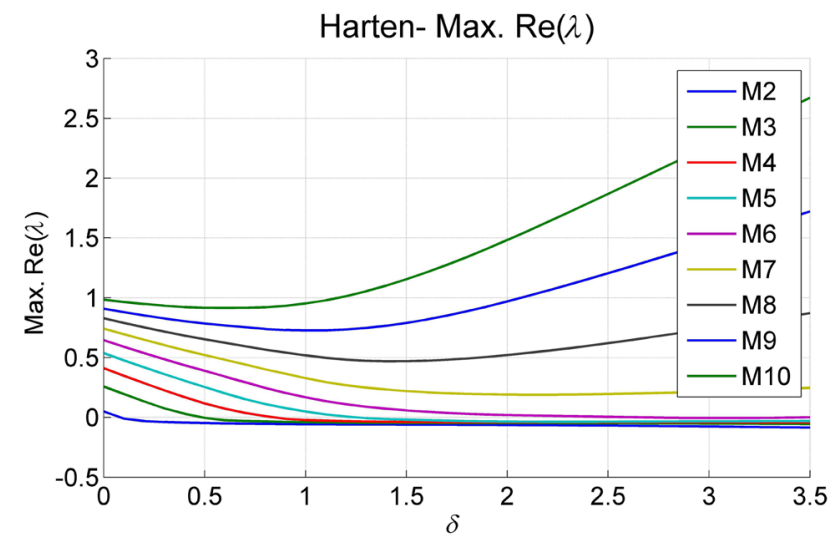

Figure 8. Image showing the variation of maximum real part of eigenvalues of the stability matrix with the value of Harten's parameter for flows with Mach number 2-10.

\section{Analysis of the sonic glitch}

The sonic glitch, like the carbuncle, is an undesirable sideeffect of employing upwinding schemes. It is an unphysical expansion-shock-like jump found near the sonic points. Roe [29] studied the flux at the sonic point and suggested that apart from modifying the sonic flux one needs treatment of the neighbouring points as well. Tang [30] too performed analytical and numerical analysis on sonic glitch for Burgers' equation and argued that there is no relation between the sonic glitch and violation of entropy condition. The problem has not, however, received much attention in the literature as it can be fixed easily through a Harten fix without too many side effects. Our interest in the sonic glitch, however, stems for certain intriguing similarities it shares with the carbuncle:

1. Both the sonic glitch and carbuncle are exhibited by socalled "entropy-satisfying" schemes such as the Godunov scheme.

2. Both are corrected by Harten's fix. However, there is justification in neither case for using a finite-sized Harten parameter.

3. Both problems either disappear or become less severe on the reduction of grid size or on the increase of the order of the method.

Given these similarities, it is fruitful to consider if one can explain why a finite-sized Harten parameter is needed for the sonic-glitch, as it offers an analytically easier setting than the carbuncle. For this purpose, we perform the analysis on the scalar inviscid Burgers equation. In conservation form, this hyperbolic PDE can be written as

$$
\frac{\partial u}{\partial t}+\frac{\partial}{\partial x}\left(\frac{u^{2}}{2}\right)
$$

where $u$ is any scalar and $\frac{u^{2}}{2}$ is the flux of the scalar $u$. The sonic point is defined as the point where $\frac{\partial f}{\partial u}=0$. For
Burgers' equation, this comes out to be the point where $u=0$.

In what follows, we perform a non-linear, entropy-based analysis of the sonic glitch. Our primary contributions are that firstly, we differ from Tang [30] and show conclusively that the sonic glitch is indeed an entropy problem. Secondly, we argue that some artificial dissipations may seem dissipative but might actually be adding anti-dissipation. In the same vein, we derive a new entropy criterion that clarifies why this confusion in dissipation may arise. We finally connect this idea to that of the origins of the carbuncle.

\subsection{Sonic glitch and the Godunov scheme}

As mentioned earlier, one of the puzzling facts about the sonic glitch is that even entropy-satisfying schemes such as the Godunov scheme exhibit it. This has led to the recent conclusion (see [30]) that the glitch does not originate from any non-linear entropy violations. The issue is muddled even more by the fact that, for the Burgers equation, Roe's scheme, which does not satisfy entropy, is exactly identical to Godunov, except at the sonic point. However, as Roe [29] shows, fixing the sonic point alone does not fix the sonic glitch, leading to a paradoxical situation.

To clarify the situation, we consider the Godunov scheme, which computes fluxes through the exact solution of the Riemann problem at every cell face.

For a Riemann problem of the form

$$
u_{0}(x)= \begin{cases}u_{L} & \text { if } x<0 \\ u_{R} & \text { if } x>0\end{cases}
$$

where $u_{L}$ and $u_{R}$ are constants, the exact solution is obtained by considering the two cases possible depending on the values of $u_{L}$ and $u_{R}$ :

1. if $u_{L}>u_{R}$, a shock wave is formed with shock speed $s$ satisfying $f\left(u_{R}\right)-f\left(u_{L}\right)=s\left(u_{R}-u_{L}\right)$ That is, a shock propagates at $s=\frac{u_{R}+u_{L}}{2}$. The solution at any time $t$ is then given by

$$
u(x, t)= \begin{cases}u_{L} & \text { if } x<s t \\ u_{R} & \text { if } x>s t\end{cases}
$$

2. if $u_{L}<u_{R}$, an expansion fan (rarefaction wave) is formed and the solution at any time $t$ is then given by

$$
u(x, t)=\left\{\begin{array}{cl}
u_{L} & \text { if } x<u_{L} t \\
\frac{x}{t} & \text { if } u_{L} t \leq x \leq u_{L} t \\
u_{R} & \text { if } x>u_{R} t
\end{array}\right.
$$

However, despite using the above exact Riemann solution at the flux faces, simulation of an expansion with $u_{L}=-1$ and $u_{R}=1$ using Godunov scheme results in a glitch that 


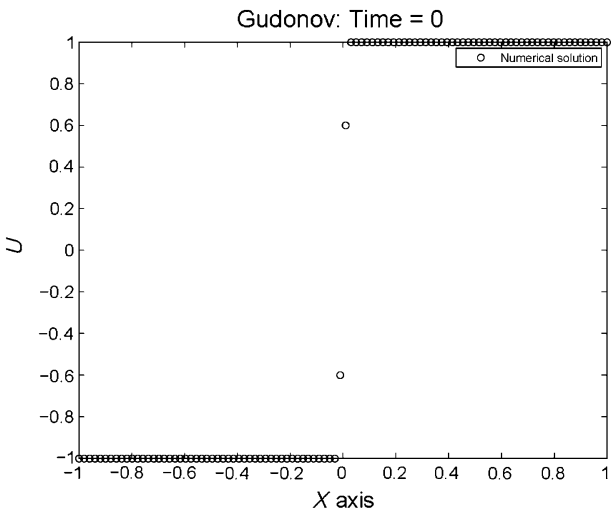

(a)

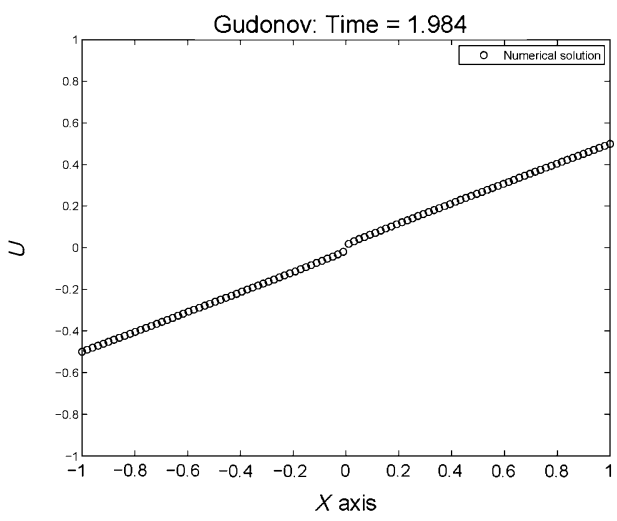

(c)

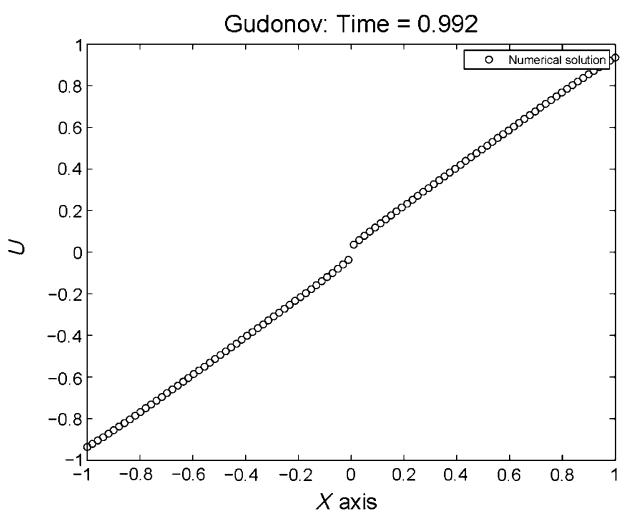

(b)

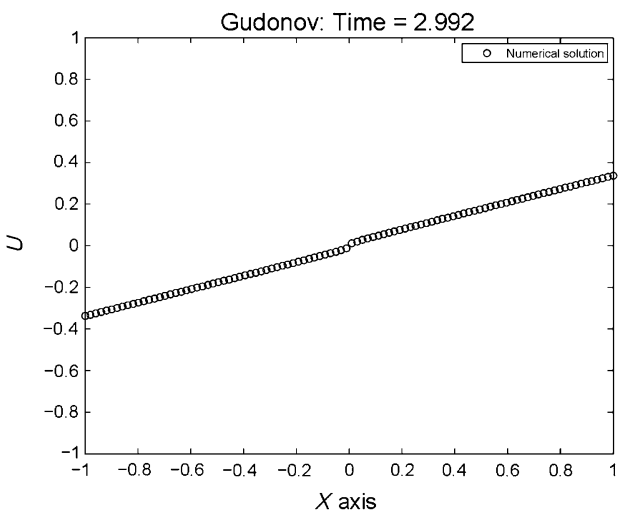

(d)

Figure 9. Figure showing the evolution of numerical solution with time computed using Godunov's scheme. Figure (a) shows the initial conditions and the subsequent figures show solution at different times. The glitch can be clearly seen in the plots. (a) Godunov scheme, $t=0 \mathrm{~s}$. (b) Godunov scheme, $t=1 \mathrm{~s}$. (c) Godunov scheme $\mathrm{t}=2 \mathrm{~s}$. (d) Godunov scheme, $t=3 \mathrm{~s}$.

looks like an expansion shock right around the sonic point. Figure 9 shows the numerical solution computed using Godunov scheme at four different time steps. Note that the actual solution is a smooth rarefaction wave but the Godunov solution shows the points near the sonic point $x=0$ exhibiting an expansion-shock-like behaviour. A similar behaviour is also exhibited by Roe's scheme with an insufficiently large value of Harten's fix.

Analysis of the entropy jump across the sonic point using the standard definition of entropy (see [31]) confirms that there is no entropy violation across the sonic point when we use the Godunov scheme. What then is the origin of the glitch and the resolution of the paradox? We resolve this issue by showing that the sonic glitch is indeed an entropy violation and that the definition of numerical entropy generation needs to derived carefully.

\subsection{The sonic glitch and entropy violation}

One can relate the entropy properties of a scheme to its dissipative nature. Intuitively, a scheme satisfying entropy condition would have at least as much dissipation as that of the exact solution. In other words, having lesser dissipation than that of the exact solution would be a violation of the entropy condition. In the case of the expansion fan, the relative amount of dissipation can easily be discerned from the rate at which the solution decays, i.e., how rapidly the slope decays.

If, therefore, we believe that numerical schemes like Roe and Godunov satisfy entropy and hence add adequate dissipation near the sonic point, the rate of decay of the numerical gradient of numerical schemes would be greater than the exact rate of decay of gradient. Violation of this criterion would be tantamount to violation of entropy. We now check if this is true.

Assuming the domain to be divided into uniform partition of size $h$ and that the sonic point $(u(x=0)=0)$ has the index $i$ (see figure 10), the analysis is done for the sonic point and its neighbouring points to show the discrepancy in the numerical schemes.

The rate of decay of gradient, i.e., $u_{x t}$ is analysed. As the actual solution is smooth, the rate of dissipation of gradient can be easily found by simply taking the partial derivative with respect to time and space. The analysis is being done at a time $t_{n}$; therefore the actual rate of dissipation of gradient is given as 


$$
\left.u_{x t}\right|_{t_{n}}=\left\{\begin{array}{cl}
0 & \text { if } x(i)<-t_{n} \\
-\frac{1}{t_{n}^{2}} & \text { if }-t_{n} \leq x(i) \leq t_{n} . \\
0 & \text { if } x(i)>t_{n}
\end{array}\right.
$$

The actual solution of the Riemann problem for the given discretization with given initial conditions at time $t_{n}$ can be given as

$$
u_{i}^{n}=\left\{\begin{array}{cl}
-1 & \text { if } x(i)<-t_{n} \\
\frac{x(i)}{t_{n}} & \text { if }-t_{n} \leq x(i) \leq t_{n} . \\
1 & \text { if } x(i)>t_{n}
\end{array}\right.
$$

It is assumed that this is the initial condition; solution is developed from this point on and the time step is $\tau$. The rate of dissipation of gradient provided by Roe and Godunov scheme can now be computed.

3.2a Decay rate of Godunov scheme: Godunov scheme uses the exact solution of Riemann problem at the cell interface. The flux at the interface in Godunov scheme is given as

$$
f_{i+\frac{1}{2}}^{\text {Godunov }}=f^{\text {Godunov }}\left(u_{i}, u_{i+1}\right)=\max \left\{\frac{1}{2}\left(u_{i}^{+}\right)^{2}, \frac{1}{2}\left(u_{i+1}^{-}\right)^{2}\right\},
$$

where $u^{+}=\max (u, 0)$ and $u^{-}=\min (u, 0)$. Therefore the solution obtained at $t_{n}+\tau$ using the Godunov scheme is

$$
u_{i}^{n+1}=0, u_{i+1}^{n+1}=\frac{h}{t_{n}}-\frac{\tau h}{2 t_{n}^{2}}, u_{i-1}^{n+1}=-\frac{h}{t_{n}}+\frac{\tau h}{2 t_{n}^{2}} .
$$

Using the central difference scheme (CDS) to find the gradient and then using the forward difference scheme to find the rate of change of gradient, we obtain

$$
\begin{aligned}
\left(u_{i}^{n}\right)_{x}^{\text {Godunov }} & =\frac{u_{i+1}^{n}-u_{i-1}^{n}}{2 h}=\frac{1}{t}, \\
\left(u_{i}^{n+1}\right)_{x}^{\text {Godunov }} & =\frac{u_{i+1}^{n+1}-u_{i-1}^{n-1}}{2 h}=\frac{1}{t}-\frac{\tau}{2 t^{2}}, \\
\left(u_{i}^{n}\right)_{x t}^{\text {Godunov }} & =\frac{\left(u_{i}^{n+1}\right)_{x}^{\text {Godunov }}-\left(u_{i}^{n}\right)_{x}^{\text {Godunov }}}{\tau}=-\frac{1}{2 t_{n}^{2}} .
\end{aligned}
$$

Similarly, the rate of dissipation of the gradient at the neighbouring cells can be computed. The result is as follows:

$$
\begin{aligned}
\left(u_{i}^{n}\right)_{x t}^{\text {Godunov }} & =-\frac{1}{2 t_{n}^{2}}, \\
\left(u_{i+1}^{n}\right)_{x t}^{\text {Godunov }} & =-\frac{3}{4 t_{n}^{2}}=\left(u_{i-1}^{n}\right)_{x t}^{\text {Godunov }}, \\
\left(u_{i+2}^{n}\right)_{x t}^{\text {Godunov }} & =-\frac{5}{4 t_{n}^{2}}=\left(u_{i-2}^{n}\right)_{x t}^{\text {Godunov }} .
\end{aligned}
$$

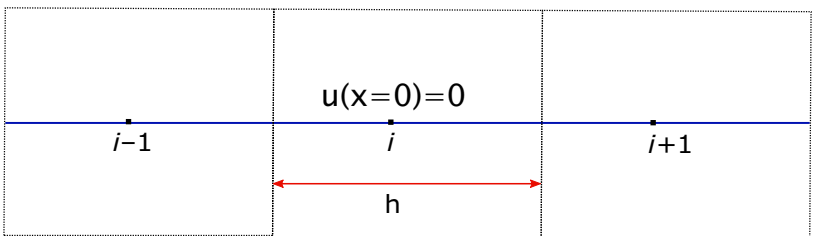

Figuer 10. Image showing the grid defined for the analysis; $h$ is the grid size and index $i$ is given to the sonic point.

The results show clearly that Godunov scheme dissipates lesser than the exact solution near the sonic point. However, as we move away from the sonic point, the rate of dissipation becomes greater than the actual. This mirrors the behaviour of the sonic glitch exactly but it leads to the surprising conclusion that the Godunov scheme violates entropy at the sonic point. There is one further surprise in store. A quick check shows that for a smooth expansion, the CDS gives the exact rate of decay but the Godunov scheme decays at a slower rate near the sonic point. This leads to the conclusion that, in certain cases, the Godunov scheme is less dissipative that the central scheme, which is not dissipative at all. That is, the Godunov scheme can actually add anti-dissipation.

3.2b Decay rate of Roe's scheme: Roe's scheme approximately solves the Riemann problem at the cell interface and the flux in Roe's scheme is given by

$$
f_{i+\frac{1}{2}}^{R o e}=f^{R o e}\left(u_{i}, u_{i+1}\right)=\frac{1}{2}\left(f\left(u_{i}\right)+f\left(u_{i+1}\right)\right)-\frac{1}{2} \mid \lambda_{i+\frac{1}{2}}\left(u_{R}-u_{L}\right)
$$

where $\left|\lambda_{i+\frac{1}{2}}\right|=\frac{f\left(u_{R}\right)-f\left(u_{L}\right)}{u_{R}-u_{L}}$ is the characteristic speed evaluated at the cell interface. However, note that Roe's scheme admits non physical expansion shocks and Harten's entropy fix is required to cure the problem. We therefore analyse the Roe-H scheme. A similar procedure is carried out as before and the solution at next time step is found using Roe's scheme. It is assumed that the Harten's fix is employed till cell $i+2$, after which the characteristic speed is sufficiently high to add proper dissipation. The dissipation rate of gradient, assuming the value of Harten's parameter to be $\delta$ for the cells near the sonic point, is

$$
\begin{aligned}
\left(u_{i}^{n}\right)_{x t}^{R o e} & =-\frac{1}{t_{n}^{2}}+\frac{h}{2 \delta t_{n}^{3}}, \\
\left(u_{i+1}^{n}\right)_{x t}^{R o e} & =-\frac{1}{t_{n}^{2}}+\frac{h}{2 \delta t_{n}^{3}}=\left(u_{i-1}^{n}\right)_{x t}^{R o e}, \\
\left(u_{i+2}^{n}\right)_{x t}^{R o e} & =-\frac{3}{2 t_{n}^{2}}-\frac{17 \tau h}{32 \delta t_{n}^{3}}-\frac{\delta}{8 h t_{n}}=\left(u_{i-2}^{n}\right)_{x t}^{R o e}, \\
\left(u_{i+3}^{n}\right)_{x t}^{R o e} & =-\frac{3}{2 t_{n}^{2}}-\frac{h}{2 \delta t_{n}^{3}}=\left(u_{i-3}^{n}\right)_{x t}^{R o e} .
\end{aligned}
$$


The results are similar to those of Godunov scheme. The rate of dissipation of gradient is less than the actual near the sonic point and is greater away from it. This means that the numerical dissipation is less than actual dissipation by $\frac{h}{2 \delta t_{n}^{3}}$. Thus the numerical dissipation will get close to actual dissipation if the grid size is decreased or Harten's parameter is increased. Both of these results are supported by numerical experiments. The analysis also confirms that the glitch is located on and near the sonic point, as away from the sonic point the conditions as defined are satisfied. Further, we have succeeded in explaining why a finite Harten parameter is needed for entropy satisfaction.

We would like to also note here some comments about the case when sonic points are completely absent. In such a case, Roe and Godunov schemes (and by extension LRS also) become completely equivalent if no sonic point is present in the solution. In the case where sonic point is not present in the rarefaction wave, the rate of dissipation of gradient is equal for the numerical and actual solution which is $-\frac{1}{t^{2}}$.

We summarize the conclusions of the above analysis. Firstly, it is clear that the Godunov and Roe-H schemes (with an inadequate Harten parameter) have insufficient dissipation near and around the sonic point causing the glitch. Secondly, the origins of the finite Harten parameter stem from the fact that the actual dissipation addition might be insufficient. Thirdly, although both schemes add diffusion to the fluxes, the resulting dissipation might be insufficient or, in some cases, there may evenbe some anti-dissipation! While this analysis qualitatively accounts for the sonic glitch, it still does not quantify the amount of entropy violation. We now reinterpret and modify existing entropy definitions and analyse the entropy behaviour of Roe's scheme, Godunov scheme (LRS scheme) and the unstable CDS.

\subsection{Modified entropy analysis}

With weak formulation of hyperbolic conservation laws problems with discontinuities do not always have unique solutions. In order to identify the correct, physical solutions, the second law of thermodynamics has to be enforced apart from the conservation laws. The general formulation of the entropy condition for hyperbolic conservation laws was first given by Lax [32] and is given by the equation

$$
\bar{E}_{t}+\bar{F}_{x} \leq 0
$$

where $\bar{E}$ is the entropy and $\bar{F}$ is its flux and the pair is such that

$$
\bar{E}_{u u}<0 \bar{F}_{u}=\bar{E}_{u} f_{u},
$$

where $f$ and $u$ are defined as usual. For scalar conservation equations such as Burgers' equation, one can define $E=$ $-u^{2}$ as the second law of thermodynamics states the entropy should increase for a closed system. However from the mathematical point of view, instead of an increasing function, it can be defined to be an decreasing function on which an upper bound can be set. Therefore, in this study, $u^{2}$ will be defined as the entropy. We note that this entropy can also be interpreted as the energy, a fact we will exploit in the following.

Merriam [31] carried out an analysis to satisfy the entropy inequality cell by cell and devised an entropy-satisfying scheme based on this. However, Merriam's criterion does not detect the sonic glitch as an entropy violation. Here, an attempt is made to reinterpret this cell by cell entropy inequality by exploiting the fact that, for scalar conservation laws, entropy and energy are equivalent. Based on this reinterpretation, we extend Merriam's version of entropy inequality.

Merriam's inequality proceeds from Eq. (31). On applying it to a given grid point, one obtains

$$
2 u_{i} u_{t}+\frac{F_{i+1 / 2}-F_{i-1 / 2}}{h} \leq 0,
$$

where Merriam suggests that $u_{t}=-f_{x}$ be computed from the satisfaction of the conservation law using the numerical flux. For Godunov's method, at the sonic point of an expansion, $u_{t}=0$ and $+\frac{F_{i+1 / 2}-F_{i-1 / 2}}{h}=0$ using the exact Riemann solver. Hence, the entropy inequality appears to be satisfied, which is incorrect.

We reinterpret Merriam's inequality (32) as follows. The first term is the numerical rate of change of energy. The second term is the negative of the exact rate of change of energy. This is seen easily by integrating Eq. (31) in space. Hence, Merriam's inequality can be interpreted as follows;

$$
\dot{E}_{\text {num }} \leq \dot{E}_{\text {exact }} .
$$

That is, the numerical rate of change of energy in any domain is not greater than the exact rate of change of energy. Interpreting the inequality in this fashion clarifies why this inequality determines local unphysical behaviour; pumping in of numerical energy faster than the rate dictated by the equation leads to spurious energy being distributed in the domain. This would be analogous to adding antidissipation.

We now employ this interpretation in determining a better entropy equation for scalar conservation laws. We find the rate of change of entropy/energy contained between the cells $i$ and $i+1$. Consider two cells having the cellaveraged values $u_{L}$ and $u_{R}$ with grid-size $h$. Distance between the two cell centres would be $h$ if the grids are uniform. Figure 11 presents the case.

The numerical and the actual rate of change of total entropy contained between the two cells need to be expressed. First, starting with the Burgers equation, we multiply the equation by $2 u$ to get 


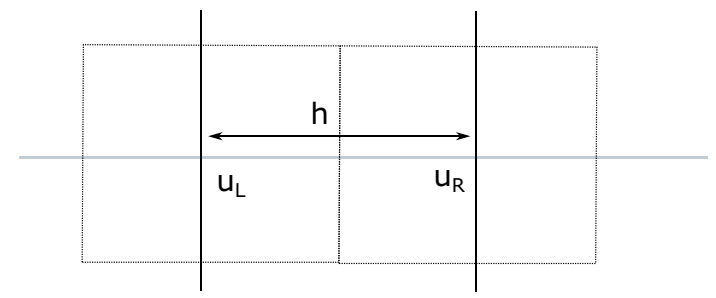

Figure 11. Image showing two cells having $u_{L}$ and $u_{R}$ as the cellaveraged value with $h$ as the grid size.

$$
2 u u_{t}+2 u^{2} u_{x}=0, \Rightarrow\left(u^{2}\right)_{t}+\left(\frac{2}{3} u^{3}\right)_{x}=0
$$

To find the rate of change of entropy between the two cells, integrate this equation between the cell centres, i.e.

$\int_{0}^{h}\left(\left(u^{2}\right)_{t}+\left(\frac{2}{3} u^{3}\right)_{x}\right) d x=0\left(\int_{0}^{h}\left(u^{2}\right) d x\right)_{t}=-\int_{0}^{h}\left(\frac{2}{3} u^{3}\right)_{x} d x=0$

$\int_{0}^{h}\left(u^{2}\right) d x$ is the total entropy between the two cells. Therefore, finally the following relation is obtained for the actual rate of change of entropy:

$$
\dot{E}_{\text {exact }}=\frac{2}{3}\left(u_{L}^{3}-u_{R}^{3}\right)
$$

For numerical rate of change of entropy, assume the scalar $u$ to be varying linearly between the two cells. This linear variation is the essential difference between our formulation and others that exist in the literature. In the linear case, the value of $u$ at any intermediate point can be given as

$$
u(x)=\frac{u_{R}-u_{L}}{h} x+u_{L} .
$$

Now, integrate the entropy between the cells:

$$
E_{n u m}=\int_{0}^{h} u(x)^{2} d x=\int_{0}^{h}\left(\frac{u_{R}-u_{L}}{h} x+u_{L}\right)^{2} d x .
$$

After integration, differentiate the net entropy with respect to time to get the following equation:

$$
\dot{E}_{n u m}=\frac{h}{3}\left(\dot{u}_{L}\left(2 u_{L}+u_{R}\right)+\dot{u}_{R}\left(u_{L}+2 u_{R}\right)\right)
$$

$\dot{u}_{L}$ and $\dot{u}_{R}$ are the rate of change of $u$, which can be found using the scalar conservation equation (23). Note that the rate of change of the quantity would be equal to negative of the gradient of flux, which can be computed through the scheme employed. Using Eqs. (34) and (35), the actual rate of change of entropy and the numerical rate of change of entropy for Roe's scheme, Godunov scheme and CDS for the actual solution at a certain time $t$ (same form of analysis as done before) is calculated and is given in table 1

We note the following features that our theory exhibits, which are completely consistent with numerical experiments:

1. The actual and the numerical rate of change of entropy for all the schemes away from the rarefaction wave (i.e., $x>t$ and $x<-t$ ) are equal to zero and thus satisfy the condition (33).

2. The entropy condition is not satisfied at the sonic point as well as it its neighbours which explains Roe's result in Roe [29] that one needs to fix both the sonic point as well as its neighbours. To our knowledge, ours is the only non-ad-hoc explanation of Roe's observation.

3. For the Godunov scheme, the numerical rate of change of entropy approaches the actual one as one moves away from the sonic point, reinforcing that the flaw is in the treatment of sonic point and its neighbouring points.

4. For the Roe scheme again, the difference between actual and numerical rate of change of entropy is dependent on a high order of grid-size, meaning if $h \rightarrow 0$ the numerical and actual entropy change rate will become equal, i.e., on decreasing the grid size the error should reduce.

5. One can also observe the dependence of the entropy condition on Harten's parameter. If the Harten's parameter is large, then the numerical and actual entropy change rate would become almost equal. Once again, the dependence of entropy violations on the size of Harten's parameter has not been found in the literature.

6. The greatest surprise is in the result that, for the CDS, the actual and numerical rates of change of entropy are equal. As with our results in section 3.2a, this indicates that the effective artificial viscosities of the Godunov

\begin{tabular}{|c|c|c|c|c|}
\hline Cells & RoCoE actual & RoCoE CDS & RoCoE Godunov & RoCoE Roe \\
\hline$i \rightarrow i+1$ & $-\frac{2}{3}\left(\frac{h}{t}\right)^{3}$ & $-\frac{2}{3}\left(\frac{h}{t}\right)^{3}$ & $-\frac{1}{3}\left(\frac{h}{t}\right)^{3}$ & $-\frac{2}{3}\left(\frac{h}{t}\right)^{3}+\frac{2}{3} \frac{h^{4}}{2 \delta t^{4}}$ \\
\hline$i+1 \rightarrow i+2$ & $-\frac{2}{3}(7)\left(\frac{h}{t}\right)^{3}$ & $-\frac{2}{3}(7)\left(\frac{h}{t}\right)^{3}$ & $-\frac{2}{3}\left(\frac{19}{4}\right)\left(\frac{h}{t}\right)^{3}$ & $-\frac{2}{3}(7)\left(\frac{h}{t}\right)^{3}+\frac{2}{3}(7) \frac{h^{4}}{2 \delta t^{4}}$ \\
\hline$i+2 \rightarrow i+3$ & $-\frac{2}{3}(19)\left(\frac{h}{t}\right)^{3}$ & $-\frac{2}{3}(19)\left(\frac{h}{t}\right)^{3}$ & $-\frac{2}{3}\left(\frac{61}{4}\right)\left(\frac{h}{t}\right)^{3}$ & $-\frac{2}{3}(19)\left(\frac{h}{t}\right)^{3}+\frac{14}{3}\left(\frac{h}{t}\right)^{3}-\frac{2}{3} \frac{11 h^{4}}{4 \delta t^{4}}-\frac{2}{3} \frac{\delta h^{2}}{t^{2}}$ \\
\hline$i+3 \rightarrow i+4$ & $-\frac{2}{3}(37)\left(\frac{h}{t}\right)^{3}$ & $-\frac{2}{3}(37)\left(\frac{h}{t}\right)^{3}$ & $-\frac{2}{3}\left(\frac{127}{4}\right)\left(\frac{h}{t}\right)^{3}$ & $-\frac{2}{3}(37)\left(\frac{h}{t}\right)^{3}+\frac{23}{3}\left(\frac{h}{t}\right)^{3}-\frac{2}{3} \frac{125 h^{4}}{16 \delta t^{4}}-\frac{2}{3} \frac{4 \delta h^{2}}{t^{2}}$ \\
\hline$i+4 \rightarrow i+5$ & $-\frac{2}{3}(61)\left(\frac{h}{t}\right)^{3}$ & $-\frac{2}{3}(61)\left(\frac{h}{t}\right)^{3}$ & $-\frac{2}{3}\left(\frac{217}{4}\right)\left(\frac{h}{t}\right)^{3}$ & $-\frac{2}{3}(61)\left(\frac{h}{t}\right)^{3}+\frac{2}{3}\left(\frac{27}{4}\right)\left(\frac{h}{t}\right)^{3}$ \\
\hline
\end{tabular}
and Roe schemes are anti-dissipative (near the sonic point).

Table 1. Table containing the values of actual rate of change of entropy and numerical rate of change of entropy of Roe and Godunov schemes and CDS. Note: RoCoE-rate of change of entropy. 
In summary, the results of this section show that, in contrast to some results in the literature, it is possible to describe the sonic glitch as an entropy violation through a novel entropy condition. Further, the same definition also offers an explanation for why a finite Harten's parameter is required for fixing the sonic glitch.

\section{Conclusion}

A thorough stability analysis of the simplified carbuncle was performed. All possible modes of linear instability were considered, including non-modal ones. While the matrix stability analysis matched well with the numerical experiments in most cases, it was also unable to explain several features. Firstly, modal and non-modal stability analysis showed that even a marginally positive value of Harten's parameter could cure the carbuncle, which was in direct disagreement with the numerical simulations. Secondly, the analysis also showed similar behaviour for the Roe and LRS schemes while there are significant qualitative differences between the two schemes. The failure of even non-modal analysis implies that non-normality is not the issue causing the carbuncle even though the stability matrices obtained after linearizing the problem are highly non-normal. We conclude therefore that there is an essentially non-linear mechanism behind the carbuncle.

In order to analyse the nature of this mechanism, we investigated the sonic glitch for Burger's equation using an entropy-based non-linear stability analysis. This case was chosen as Harten's fix exhibits similar features in this as well as the carbuncle problem. Through a modified version of the entropy inequality, we proved that the sonic glitch appears due to a local entropy violation. We also showed that the artificial viscosities in Roe and Godunov schemes can sometimes dissipate even less than the nondissipating CDS and can hence be anti-dissipative in nature. We conjecture that a similar, non-linear, anti-dissipation mechanism may be in operation in the carbuncle instability. The current theory is inadequate to determine this as the modified entropy definition applies only to scalar equations. In order to test the non-linear theory on the actual carbuncle case, therefore, the non-linear entropy inequality needs to be extended to systems of non-linear equations. Work on this is currently in progress and we shall report future developments in a forthcoming publication.

\section{References}

[1] Quirk J J 1997 A contribution to the great Riemann solver debate.Springer

[2] Ismail F 2006 Toward a reliable prediction of shocks in hypersonic flow: resolving carbuncles with entropy and vorticity control. Ph.D. Thesis, University of Michigan, Ann Arbor

[3] Kitamura K, Roe P and Ismail F 2009 Evaluation of Euler fluxes for hypersonic flow computations. AIAA J. 47(1): 44-53

[4] Kudriakov S and Hui W 2008 On a new defect of shockcapturing methods. J. Comput. Phys. 227(3): 2105-2117

[5] Roe P, Nishikawa H, Ismail F and Scalabrin L 2005 On carbuncles and other excrescences. AIAA Paper 4872

[6] Peery K and Imlay S 1988 Blunt-body flow simulations. AIAA Paper 2904

[7] Garicano-Mena J, Lani A and Deconinck H 2016 An energydissipative remedy against carbuncle: application to hypersonic flows around blunt bodies. Comput. Fluids 133: 43-54

[8] MacCormack R W 2011 The carbuncle CFD problem. In: Proceedings of the 49th AIAA Aerospace Sciences Meeting, pp. 2011-381

[9] Kitamura K and Shima E 2013 Towards shock-stable and accurate hypersonic heating computations: a new pressure flux for AUAM-family schemes. J. Comput. Phys., 245: 62-83

[10] Pandolfi M and D'Ambrosio D 2001 Numerical instabilities in upwind methods: analysis and cures for the carbuncle phenomenon. J. Comput. Phys. 166(2): 271-301

[11] Powers J M, Bruns J D and Jemcov A 2015 Physical diffusion cures the carbuncle phenomenon. In: Proceeding of the 53rd AIAA Aerospace Sciences Meeting, p. 0579

[12] Elling V 2009 The carbuncle phenomenon is incurable. Acta Math. Sci. 29(6): 1647-1656

[13] Harten A 1983 High resolution schemes for hyperbolic conservation laws. J. Comput. Phys. 49(3): 357-393

[14] Dumbser M, Moschetta J M and Gressier J 2004 A matrix stability analysis of the carbuncle phenomenon. J. Comput. Phys. 197(2): 647-670

[15] Liou M S 2000 Mass flux schemes and connection to shock instability. J. Comput. Phys. 160(2): 623-648

[16] Kim S S, Kim C, Rho O H and Kyu Hong S 2003 Cures for the shock instability: development of a shock-stable roe scheme. J. Comput. Phys. 185(2): 342-374

[17] Robinet J C, Gressier J Casalis G and Moschetta J M 2000 Shock wave instability and the carbuncle phenomenon: same intrinsic origin? J. Fluid Mech. 417: 237-263

[18] Srinivasan B, Jameson A and Krishnamoorthy S 2012 An upwinded state approximate Riemann solver. Int. J. Num. Methods Fluids 70(5): 578-602

[19] Pullin D 1980 Direct simulation methods for compressible inviscid ideal-gas flow. J. Comput. Phys. 34(2): 231-244

[20] Macrossan M and Oliver R 1993 A kinetic theory solution method for the Navier-Stokes equations. Int. J. Num. Methods Fluids 17(3): 177-193

[21] Gressier J and Moschetta J M 2000 Robustness versus accuracy in shock-wave computations. Int. J. Num. Methods Fluids 33(3): 313-332

[22] Sanders R, Morano E and Druguet M C 1998 Multidimensional dissipation for upwind schemes: stability and applications to gas dynamics. J. Comput. Phys. 145(2): 511-537

[23] Gustavsson L H 1991 Energy growth of three-dimensional disturbances in plane Poiseuille flow. J. Fluid Mech. 224: 241-260

[24] Butler K M and Farrell B F 1992 Three-dimensional optimal perturbations in viscous shear flow. Phys. Fluids A: Fluid Dyn. 4(8): 1637-1650 
[25] Reddy S C, Schmid P J and Henningson D S 1993 Pseudospectra of the Orr-Sommerfeld operator. SIAM J. Appl. Math. 53(1): 15-47

[26] Reddy S C and Henningson D S 1993 Energy growth in viscous channel flows. J. Fluid Mech. 252: 209-238

[27] Trefethen L N, Trefethen A, Reddy S, Driscoll T et al 1993 Hydrodynamic stability without eigenvalues. Science 261(5121): 578-584

[28] Wright T G 2002 Eigtool. http://www.comlab.ox.ac.uk/pseu dospectra/eigtool/
[29] Roe P 1992 Sonic flux formulae. SIAM J. Sci. Stat. Comput. 13(2): 611-630

[30] Tang H 2005 On the sonic point glitch. J. Comput. Phys. 202(2): 507-532

[31] Merriam M L 1989 An entropy-based approach to nonlinear stability. NASA Technical Memorandum 101086(64): p. 1

[32] Lax P D 1973 Hyperbolic systems of conservation laws and the mathematical theory of shock waves, vol. 11. SIAM 